\title{
EDUCAÇÃO ON-LINE EM CONTABILIDADE: PROPENSÃO E ASPECTOS CURRICULARES ${ }^{1}$
}

\section{ON-LINE ACCOUNTING EDUCATION: TENDENCY AND CURRICULUM ASPECTS}

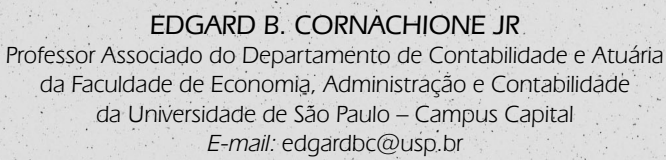

EDGARD B. CORNACHIONE JR

Professor Associado do Departamento de Contabilidade e Atuária da Faculdade de Economiă, Administraçà̀o e Contabilidàde da Universidade de Săo Paulo - Campus Capital E-mail::edgardbc@usp.br

\author{
SILVIA PEREIRA DE CASTRO CASA NOVA \\ Professora Doutora do" Departàmento de Contabilidade e Atuária \\ da Faculdade de Economia, Administração e Contabilidade \\ da Universidade de São Paulo - Campus Capital \\ E-mạil: silvianova@usp.br
}

\author{
MARIA ROSA TROMBETTA \\ Mestranda em Controladoria e Contabilidade \\ pela Faculdade de Economia, Administração e Contạbilidade \\ da Universidade de São Paulo - Campus Capital \\ E-mail: rosa.trombetta@fipecafi.org
}

\section{RESUMO}

Com base na literatura envolvendo educação na área de negócios, bem como nos achados de pesquisadores da área de desenvolvimento de recursos humanos e considerando as necessidades educacionais e de treinamento no ambiente de trabalho, este estudo foca a análise da metacognição e propensão de estudantes-executivos (alunos adultos trabalhadores) em participar de disciplinas oferecidas on-line relacionadas a programas de MBA com ênfase em Contabilidade e áreas afins, além de explorar quais conteúdos específicos podem estar relacionados a esse fenômeno. $\mathrm{O}$ crescimento da oferta de cursos on-line, considerando a flexibilidade e adaptabilidade que essa modalidade de ensino permite, reforça a importância da compreensão dos fatores que influenciam a propensão à participação: Segundo dados do Anuário Brasileiro Estatístico de EAD 2007, um em cáda 80 brasileiros estudou por EAD em 2006. Foram cerca 2.279.000 alunos a distância matriculados em vários tipos (ensino credenciado, educação corporativa e em outros projetos nacionais e regionais) dentre os 889 cursos a distância (credenciados pelo Sistema de Ensino - MEC e conselhos estaduais de educação). Esses números confirmam a importância de se examinar esse tema. Foi estudada uma amostra composta de 189 alunos de pós-graduação matriculados em programas de MBA em Contabilidade, no formato presencial, oferecido por reconhecida instituição brasileira de ensino e pesquisa (a Fundação Instituto de Pesquisas Contảbeis, Atuariais e Financeiras - FIPECAFI, órgão de apoio ao Departamento de Contabilidade e Atuária da FEA/USP). Além da análise estatística descritiva, estudos e testes de correlação, de comparação de médias e análise de conglomerados foram conduzidos para apoiar a discussão e resposta às questões de pesquisa aqui estabelecidas. De forma integrada, os resultados reforçam a premissa de que o conteúdo de disciplinas possui papel relevante na propensão dos estudantes em participar de cursos on-line. Implicações para as dimensões acadêmicas e profissionais, assim como sugestões para futuras pesquisas são também apresentadas.

Palavras-chave: MBA On-line. Ensino em Contabilidade. Currículo.

\section{ABSTRACT}

Based on business education literature, findings from human resource development scholars, and considering educational and training needs in the workplace, this study focuses on analyzing executive students' (working adults students) metacognition and tendency to attend on-line MBA courses in accounting (mainly Accounting and related subjects), and whether specific content areas can be related to this phenomenon. The increasing offer of on-line courses, based on the flexibility and adaptability granted by this education mode, reinforces the relevance of understanding the factors that influence tendencies towards students' participation. According to the 2007 Brazilian Distance Education Statistical Yearbook, one out of 80 Brazilian students relied on distance education in 2006. About 2,279,000 students enrolled in various types (e.g., accredited programs, corporate education) of 889

Recebido em 21.10.2006 • Aceito em 23.03.2007 • 2a versão aceita em 23.05.2007

Artigo originalmente apresentado na International Research Conference da Academy of Human Resource Development, 2006, Columbus, Ohio (USA)./Article originally presented at the International Research Conference of the Academy of Human Resource Development, 2006, Columbus, Ohio (USA). 
distance courses (accredited by the Ministry of Education and State Education Councils). These figures confirm the relevance of examining this topic. The sample involved 189 graduate students enrolled in a top-ranked faceto-face Brazilian accounting MBA program, offered by a renowned Brazilian education and research institution (Institute of Accounting, Actuarial and Financial Research Foundation - FIPECAFI, an organization that supports the Department of Accounting and Actuarial Science at FEA/USP). Besides descriptive statistical analysis, studies and correlation tests, comparison of means and cluster analyses were developed to support the discussion and answer the proposed research questions. In general, findings support the assumption that the course content plays a significant role in terms of students' tendency towards on-line courses. Implications for academic and professional dimensions are also presented, along with suggestions for future studies.

Keywords: On-line MBA. Accounting Education. Curriculum.

\section{INTRODUCÃO}

A pesquisa em educação on-line tem sido conduzida em diversos campos profissionais e em muitas áreas acadêmicas, baseada em diferentes enfoques. Mas é imperativo enfatizar que não existem, em grande número, estudos relacionando conteúdo e educação executiva on-line (Arbaugh, 2005). Alguns achados desses estudos sobre educação on-line incluem identificação de diferenças entre procedimentos ou formatos e também estão relacionados com substância ou essência dos conteúdos (Dabbagh e Bannan-Ritland, 2005, p. 69-103). No entanto, um aspecto central a ser considerado primeiramente é que os pesquisadores atingiram um certo nível de concordância quando considerados os usos das tecnologias, sob circunstâncias específicas (e.g., enfoques síncronos e assíncronos). Esse tipo de informação é particularmente relevante ao planejar e conduzir intervenções específicas nos ambientes organizacionais (enfoque no desenvolvimento de pessoas em ambiente de trabalho). Além disso, programas MBA podem ser considerados como intervenções indiretas (ou, em alguns casos, até mesmo diretas) baseadas na dimensão de aprendizagem (um dos principais propósitos da educação executiva), por estabelecerem condições potenciais de ações com impacto mais imediato no negócio.

Instituições acadêmicas e profissionais têm consumido esforços e recursos de forma a melhor compreender o problema e alcançar as soluções que são esperadas para apoiar decisões nessa área, buscando estabelecer um novo paradigma para empresas e organizações (consumidores), e também para fornecedores da área de treinamento e desenvolvimento.

De forma mais específica, é interessante focar uma área particular da educação corporativa: a contabilidade. O aspecto interessante dessa área de educação corporativa é que carrega um corpo de teoria bem definido, com o poder de possuir profissionais presentes dentro das organizações (e.g., contadores, gerentes financeiros, auditores internos, CFOs), ou como agentes externos (e.g., auditores, consultores, peritos, pesquisadores, instrutores). Essa visão dualista das organizações impulsiona o campo da contabilidade de tal maneira que percepções e expectativas relacionadas ao treinamento e desenvolvimento (conteúdos e técnicas) dos estudantes dessa área tendem a se tornar mais exatas ao longo do tempo, em termos de auto-motivação e metacognição (Smith, 200 I).

Com base nisso, supõe-se que estudantes ligados ao ensino superior (e.g., programas de pós-graduação), combinando experiência profissional e necessidades bem definidas de desenvolvimento, representam um grupo muito específico de alunos, que requer atenção especial das instituições educacionais ou das organizações de treinamento. São chamados por alguns pesquisadores de alunos adultos trabalhadores, em um enfoque mais geral ou, mais especificamente, de estudantes-executivos e "possuem exigências educacionais especiais e preferências como flexibilidade, conveniência, acessibilidade, custos mais baixos, e... continuam capazes de conciliar suas responsabilidades profissionais e obrigações familiares" (Huang, 2005).

Assim, compreender a percepção de detalhes instrucionais desses estudantes (e.g., conteúdo do curso, materiais, técnicas, procedimentos, métodos de acesso, avaliação, trabalho em equipe) pode ajudar no planejamento de tais elementos educacionais, proporcionando uma experiência livre de surpresas para todos os atores nesses tipos de configurações de ensino superior (McKeachie, 2002, p. I40; Merriam e Caffarella, 1999, p. 208). Estar atento a esses fatores é um aspecto relevante do processo de desenvolvimento de recursos humanos (Human Resource Development, HRD), principalmente se considerada a educação de adultos e a dimensão do ambiente de trabalho (Swanson e Holton, 200 I, p. 16I-7). Outro fator relevante é que o ensino superior tem se tornado uma indústria de serviço global (Tooley, 1999, p. I I-20) e, sob a pressão da concorrência, conhecer as preferências e necessidades dos estudantes adultos é um fator-chave para o planejamento e o projeto de programas bem sucedidos e para a sustentabilidade das instituições de ensino superior. 


\subsection{Propósito e objetivo}

O principal propósito deste artigo é contribuir para o campo de estudo de pesquisa e ensino em contabilidade, com suporte da abordagem teórica da área de desenvolvimento de recursos humanos (HRD), ao coletar evidências sobre a propensão e a metacognição sobre educação on-line de executivos pós-graduandos em contabilidade vinculados à expressiva instituição de ensino e pesquisa presente no Estado de São Paulo (Brasil), a Fundação Instituto de Pesquisas Contábeis, Atuariais e Financeiras - FIPECAFI, órgão de apoio ao Departamento de Contabilidade e Atuária da FEA/USP. Estudar metacognição, percepção e propensão dos estudantes-executivos em relação ao ambiente de educação on-line é relevante para planejar novos cursos, sendo também uma forma de feedback a cursos já iniciados, apoiando ações em termos de prover uma solução de treinamento e desenvolvimento adequada.

Assim, este artigo objetiva identificar e analisar alunos de pós-graduação matriculados em um programa de MBA em Contabilidade e áreas afins em termos de sua propensão à educação on-line, de forma a contribuir com a dimensão de treinamento e desenvolvimento de recursos humanos, atrelada à área de ensino e pesquisa em Contabilidade, enquanto busca explorar a existência de diferenças significativas em suas características e nas condições de componentes específicos do currículo (conteúdo).

\section{ARCABOUC̣O TEÓRICO}

O avanço tecnológico que tem sido experimentado atualmente, inclusive tecnologias de informação e comunicação (e.g., Internet), tem influências relevantes nos agentes sociais, em particular profissionais, instituições de ensino e organizações. Ball $(2005$, p. I) indica que "escolas de administração e negócios, assim como tantas outras instituições e programas de educação superior, lidam com a difusão das inovações da Internet em seus processos educacionais." Também as empresas buscam o benefício do emprego de soluções de treinamento corporativo apoiado por tecnologia de informação e comunicação (Gallaher, 2002). A literatura sobre educação on-line tende a ser positiva em relação aos benefícios líquidos auferidos com o emprego desta modalidade educacional, apesar de discussões sobre efeitos negativos (falhas) existirem (Noble, 2002; Romiszowski, 2004).

Bryant, Kahle e Schafer (2005) ressaltam a confusão entre os termos educação a distância (distance education) e outros como educação on-line (on-line education). Os autores chamam a atenção para os elementos que definem a educação a distância, a saber:

1. Separação quase permanente entre professor e aluno, pela duração do processo de aprendizagem;

2. Influência de organização educacional tanto no planejamento e preparo dos materiais de ensino, quanto na provisão de serviços de suporte aos alunos;

3. Uso de mídia técnica - impressa, áudio, vídeo ou computadores - para unir professor e aluno além de manter o conteúdo do curso;

4. Provisão de meios de comunicação bi-direcionais de forma que o aluno possa se beneficiar de, ou até iniciar diálogo;

5. Ausência quase permanente do grupo de alunos pela duração do processo de aprendizagem, ou seja, pessoas são normalmente tratadas como indivíduos ao invés de como grupos, com exceção de reuniões ocasionais. Tais reuniões podem ser tanto presenciais quanto por meios eletrônicos e servem a propósitos didáticos e de socialização;
Os autores, concluem, ainda, que termos tais como 'webenabled', 'on-line', e 'distributed education' focam mais especificamente nos meios pelos quais a instrução se dá, ao invés de na aprendizagem em si (Bryant, Kahle e Schafer, 2005).

A expansão da educação on-line pode ser observada em dois diferentes aspectos: o expressivo crescimento de cursos on-line administrado por instituições de ensino (Bryant, Kahle e Schafer, 2005; Gagne e Shepherd, 200 I) e o número de pesquisas realizadas na área durante os últimos anos (Bryant, Kahle e Schafer, 2005).

Certamente, a expansão foi fortemente propagada pela evolução tecnológica aliada a essa dimensão da educação a distância. O desenvolvimento de ferramentas de autoria, ambientes de aprendizagem e soluções de comunicação permite a presença de uma nova conexão de aprendizagem entre professor-aluno, aluno-aluno e aluno-conteúdo. Estudos anteriores (Arbaugh e Duray, 2002; Bryant, Kahle e Schafer, 2005; Brower, 2003; Gagne e Sheperd, 200I) tratam de fatores-chave (e.g., nível da interação, utilização de diferentes mídias, diferenças cognitivas dos estudantes, estilos de aprendizagem individuais) para a educação online, influenciando a motivação e satisfação dos alunos com a experiência de aprendizagem e, conseqüentemente, seu sucesso (Yang e Lu, 200 I).

De acordo com Bryant, Kahle e Schafer (2005), estudos tentando identificar características comuns de alunos que freqüentavam cursos on-line encontraram como resultados um público com faixa etária mais elevada, em comparação aos cursos presenciais similares, com a maioria sendo de mulheres casadas, trabalhando em tempo integral. Esse perfil está bem relacionado ao crescimento de cursos online, um elemento fundamental na aprendizagem de adultos e na educação continuada. A educação a distância é também considerada responsável por expandir os limites tradicionais da educação (e.g., universidades européias e americanas que alcançam o mercado da Ásia e do Pacífico), enfoque considerado por Huang (2005) como o movimento dos MBAs virtuais. 
A ligação do tema com o ensino de contabilidade tem sido explícita com suporte de estudos desenvolvidos com ampla variedade metodológica. Resultados estimulam o desenvolvimento de programas que se beneficiem da tecnologia de informação e comunicação, em especial Internet. Uma recente e ampla revisão de literatura sobre educação on-line (Tallent-Runnels et al., 2006), apresenta estudo com resultados de pesquisa experimental que apontam para claros benefícios advindos do emprego de modalidade on-line especificamente em curso de contabilidade, envolvendo alunos ingressantes no ensino superior. Lavoie e Rosman (2007) registram resultados do uso do modelo Research-Enriched Learning (RELM) no desenho de um programa de mestrado em contabilidade on-line. Dunbar (2004) descreve o processo exitoso de criação e conversão de um curso de contabilidade tributária para a modalidade on-line.

Mensagens positivas também são encontradas no caso específico de programas MBA oferecidos na modalidade online (Walsh, 2006). Hochberg (2006) aponta para a forte tendência de crescimento dessa modalidade instrucional em programas MBA, indicando vantagens para a nova realidade profissional, envolvendo indivíduos e organizações. Pesquisas continuam sendo desenvolvidas com foco sobre questões que, em alguns casos, ainda são perturbadoras e carecem de maior esclarecimento, como é o caso de Arbaugh e Hwang (2006) com resultados favoráveis em relação à idéa de participação do professor em programas MBA online. Complementarmente, alunos de programas MBA online podem experimentar os benefícios dessa modalidade e estudos como os de Kim, Liu e Bonk (2005) documentam precisamente tais percepções.

Retomando a questão de público, Arbaugh (2000a) indica que as principais escolas de negócios credenciadas pela $A A C S B$ perceberam que os cursos de MBA on-line podem ser atrativos para um público ainda não atendido que deseja estudar ao mesmo tempo em que trabalha. Esse tipo de curso responde, também, às necessidades corporativas específicas, envolvendo executivos bem treinados sem ter que dispor dos longos períodos de ausência usualmente necessários para programas presenciais tradicionais (Schrum e Benson, 2000). Naquela pesquisa, os alunos de um curso de MBA semi-presencial descreveram as equipes como um ponto forte das atividades on-line, enfatizando que a interação entre pares (aluno-aluno) é melhor desenvolvida em grupos menores, no quais os alunos podem estabelecer suas comunidades (Schrum e Benson, 2000; Brower, 2003). A experiência profissional pode ser também considerada como meio de promover a interação e compartilhamento de experiências dentro do grupo, baseando-se em estudos de caso reais (Brower, 2003).

Um estudo comparativo entre educação a distância e tradicional em contabilidade (Gagne e Sheperd, 200I) não encontrou diferenças no desempenho entre os alunos dos cursos on-line e dos presenciais, nem mesmo em relação à experiência de aprendizagem. Entretanto, houve diferenças significativas na avaliação do professor (feedback do estu- dante) e os alunos on-line também apontaram a interação baseada em texto (limitada em comparação à comunicação oral) como uma das fraquezas do curso on-line.

O ponto é que os fatores que afetam o desempenho dos alunos em programas on-line ainda não estão claros. Aspectos já estudados considerando o paradigma da sala de aula tradicional ainda precisam ser revistos na instrução baseada na Internet. Aspectos relevantes relacionam-se ao nível de participação e às diferenças de aprendizagem entre sexos (Arbaugh, 2000b). Também encontram-se discussões sobre o ambiente virtual como facilitador da participação de pessoas tímidas, que não se sentem confortáveis em participar ativamente de aulas tradicionais (Brower, 2003). Por outro lado, outros autores afirmam que o ambiente virtual não oferece condições para interação e isso poderia não auxiliar tais pessoas a lidarem com sua timidez (Fodor, 2003). Evidências de que os cursos on-line aumentam as interações entre os estudantes são citadas em diversos estudos e concluem que ferramentas como grupos de discussão e lista de e-mail favorecem o aumento da interação entre pares, tanto para atividades síncronas quanto assíncronas, apoiando o ambiente colaborativo de aprendizagem (Arbaugh, 2000; Brower, 2003). Alguns autores consideram que a educação a distância demandará estudantes auto-motivados e auto-didatas, portanto, estando restrita a um grupo particular de alunos (Fodor, 2003; Bryant, Kahle, Schafer, 2005), com forte metacognição.

De fato, o sucesso da educação on-line depende de diversos fatores (e.g., infra-estrutura tecnológica, tamanho da classe e experiência individual com a tecnologia), mas os professores podem atuar como facilitadores de forma a prover elementos para aumentar a participação e o envolvimento dos estudantes como meio de atingir a eficácia da educação on-line. Acompanhar as atividades dos alunos, dar feedback e trazer experiências pessoais são apenas alguns dos elementos que podem humanizar o ambiente virtual, tornando-o mais amigável e incentivando as interações (componente essencial) entre alunos.

Considerando o ensino superior e alunos adultos com experiência profissional (alunos-executivos), como é o caso de programas de MBA, uma boa parte da responsabilidade recai sobre o ombro dos próprios estudantes (Arbaugh, 2005). Dessa forma, a metacognição deve ser considerada, já que, normalmente, ajuda os alunos e outros agentes envolvidos em uma experiência de aprendizagem específica a aprimorar e enriquecer os resultados alcançados, principalmente por meio do uso de recursos e canais mais apropriados.

Alessi e Trollip (200I, p. 28) definem a metacognição como "a consciência de alguém sobre sua própria cognição". Adicionalmente, o conceito de McKeachie (2002, p. 272) sobre o processo de metacognição inclui "conhecimento de si próprio como estudante, conhecimento sobre atividades acadêmicas e conhecimento sobre estratégias a utilizar para cumprir as atividades acadêmicas." Assim, como Merrian e Caffarella (1999, p. 206) mencionam "a meta-cognição é freqüentemente vista como o mais alto 
nível de atividade mental”, dependendo de uma reflexão tão intensa que pode trazer maior acurácia ao processo, principalmente quanto à avaliação e projeção da solução.

Adicionalmente, uma revisão recente da pesquisa sobre ensino em contabilidade cita a necessidade de pesquisa em áreas que influenciem o desempenho do estudante (Paisey e Paisey, 2004; Wilson, 2002). E, em termos da dimensão organizacional (ambiente de trabalho), Cummings e Worley (2005, p. 397) estabelecem as responsabilidades dos agentes (internos e externos) em atrair e reter indivíduos altamente talentosos no ambiente de trabalho, relacionando esse enfoque ao potencial de uso de suas habilidades e conhecimentos. Dessa forma, enfrentar situações de desafio no ambiente de trabalho é uma forma relevante de estar atento às competências e habilidades (ambas, existentes ou não), e este é um direcionador natural para o reconhecimento de necessidades de treinamentos e desenvolvimentos específicos que influenciam a estrutura dos programas educacionais, como é o caso de programas de MBA.

Portanto, ao analisar programas de MBA, é desejável considerar a melhor solução para cada situação específica, envolvendo vários agentes: organização (ambiente de trabalho), alunos, professores, instituição e currículo. Esse enfoque requer conhecer as disciplinas que podem ser mais bem exploradas em ambientes virtuais ou presenciais, como também as atividades que são mais bem desenvolvidas em grupo ou individualmente (Schrum e Benson, 2000), objetivando atingir uma combinação que poderá melhor atender às características combinadas de um programa específico, instituição, alunos e professores.

Vários estudos (conforme Bryant, Kahle e Schafer, 2005) identificaram os níveis de interação requeridos por cursos de contabilidade e também propuseram considerar os estilos de aprendizagem dos alunos e professores de contabilidade, no planejamento e desenho de formatação ótima dos cursos. Recentemente, um estudo (Arbaugh, 2005) foi conduzido de forma a explicar o papel das disciplinas e conteúdos em relação à efetividade dos programas de MBA on-line. O estudo foi amplo, em termos de área, envolvendo administração de negócios como um todo. Esse enfoque dá a oportunidade e mostra o interesse de observar aspectos similares em contabilidade.

Levando tudo isso em consideração, as instituições provedoras de educação e treinamento podem ter acesso a um corpo de literatura em que experiências de pesquisa foram registradas com foco na dimensão educacional, de forma a reunir informações sobre estudantes-executivos, e isso pode contribuir para que o processo de aprendizagem como um todo torne-se mais proveitoso. Nesse aspecto particular, configurações do ambiente educacional em negócios (incluindo professores) podem também desempenhar um papel especial nesse cenário: conteúdo e processo. Maior atenção deve ser dada ao processo de aprendizagem, como forma de auxiliar os estudantes no alcance pleno de seus objetivos educacionais e profissionais.

\section{PLANEJAMENTO DA PESOUISA, QUESTÕES E LIMITAÇÕES}

A dimensão de treinamento e desenvolvimento de recursos humanos (TD, HRD) pode ser aperfeiçoada com a melhor compreensão das necessidades e condições particulares dos agentes envolvidos (e.g., indivíduos presentes no ambiente de trabalho). Nesse estudo, o principal agente é o aluno-executivo, lidando com responsabilidades profissionais enquanto busca o desenvolvimento pessoal, objetivando alavancar seu desempenho dentro dos moldes e expectativas organizacionais. Então, as questões de pesquisa aqui estabelecidas tratam dos aspectos de cursos online em uma área particular de negócios, como uma forma de contribuir com as dimensões de treinamento e desenvolvimento de recursos humanos, atrelada às dimensões de ensino e pesquisa da área de contabilidade. As questões de pesquisa apresentadas neste estudo, considerando alunos-executivos matriculados em programa MBA em Contabilidade e em áreas afins (totalizando sete diferentes cursos presenciais de MBA) oferecido por uma das principais instituições de ensino brasileiras, a Fundação Instituto de Pesquisas Contábeis, Atuariais e Financeiras - FIPECAFI, órgão de apoio ao Departamento de Contabilidade e Atuária da FEA/USP, são:

$Q_{1}$ : Em quais cursos os potenciais alunos de programas de pós-graduação em Contabilidade têm maior interesse? Quais as principais áreas de conteúdo (concentração)?

$\mathrm{Q}_{2}$ : Existem correlações significativas em termos de preferências por determinadas áreas de concentração considerando os estudantes com propensão para participar de disciplinas oferecidas on-line?

$\mathrm{Q}_{3}$ : Há diferenças significativas em termos de: (a) gênero; (b) formação acadêmica; (c) propensão a participar de disciplinas on-line em contabilidade; (d) experiência anterior em cursos on-line?

$\mathrm{Q}_{4}$ : Há diferenças significativas na propensão a participar de disciplinas on-line, nos conteúdos dos cursos e nas áreas de concentração considerando a área de concentração do curso (MBA em Controladoria vs. MBAs em áreas afins)?

$Q_{5}$. Existem agrupamentos bem definidos em termos da propensão a participar de disciplinas on-line dentre alunos de programas de pós-graduação em Contabilidade, que permitam identificar um perfil?

Para responder às questões de pesquisa este estudo não-experimental apóia-se em um enfoque de coleta de dados primários (Rea e Parker, 1997, p. 3) e em um conjunto de técnicas para coletar, registrar, compilar e analisar 
os dados, propiciando uma interpretação quantitativa dos fatos. A revisão da bibliografia embasou a análise, fornecendo fundamentos e elementos para a discussão.

Considerando que a maioria dos dados coletados, neste estudo, está relacionada à percepção de aspectos subjetivos como propensão e metacognição, coloca-se a premissa de que "não podemos ser afirmativos sobre nossas conclusões sobre o conhecimento quando estudamos o comportamento e as ações humanas" (Creswell, 2003, p. 7) como um importante delimitador quando analisados os resultados.

A pesquisa envolveu uma amostra por conveniência de alunos de pós-graduação matriculados em programas presenciais de MBA em Contabilidade e áreas afins bem-conceituados no Brasil. Os alunos estavam cursando o último semestre de 2005 e a amostra incluía tanto aqueles que estavam iniciando, quanto os que estavam em estágios mais avançados de seus respectivos programas. Todos os sujeitos assinaram um consentimento formal (com base na atual política de pesquisa com sujeitos humanos da instituição) registrando sua concordância em participar da pesquisa.

Após excluídas as respostas inválidas e considerados os termos de consentimento, a amostra conta com 189 pessoas que responderam ao instrumento com dois grupos de questões: (a) informações gerais sobre os participantes (oito itens) e (b) percepções e propensões relacionadas à educação on-line (oito itens, com um especificamente relacionado à propensão em 27 áreas de conteúdos específicos, utilizando escala Likert de cinco pontos). O instrumento de pesquisa foi estruturado com base em variáveis originais do planejamento estratégico desses programas de MBA de forma a abordar aspectos de educação on-line e foi revisado por especialistas.

A análise preliminar dos testes de confiabilidade e normalidade foi realizada para validar a qualidade dos dados, bem como para selecionar os procedimentos estatísticos mais apropriados. Além da análise estatística descritiva, estudos e testes de correlação, de comparação de médias e análise de conglomerados foram conduzidos devido à sua adequabilidade em termos das questões de pesquisa estabelecidas neste estudo, bem como da natureza dos dados coletados. Todos os tratamentos estatísticos foram desenvolvidos com o apoio do SPSS ${ }^{\odot}$, adotando nível de significância de $0.05(\alpha=0.05)$.

É importante levar em consideração as características deste estudo, em relação à amostra, e o conseqüente impacto na generalização de aspectos de seus resultados. Mesmo considerando o número de sujeitos presentes neste estudo, é crucial ponderar o ambiente cultural e social de uma economia em desenvolvimento (e.g., Brasil), assim como a área educacional particular aqui analisada (e.g., contabilidade, pós-graduação), como o método de amostragem, quando os resultados são analisados. Mas, é importante citar que, devido ao estágio da abordagem da educação contábil com esse foco na área de desenvolvimento de recursos humanos (HRD), esses resultados podem acrescentar informações e conhecimentos expressivos sobre o problema considerado por esta pesquisa.

\subsection{Coleta dos dados}

Após o teste-piloto do instrumento de pesquisa e da seleção dos programas específicos abordados por esta pesquisa, iniciou-se a coleta de dados. O processo começou pelo agendamento de uma data e horário com os professores de cada turma pesquisada. Nas datas e horários programados, os alunos receberam, primeiramente, o instrumento de pesquisa e instruções detalhadas sobre os procedimentos e objetivos sobre os itens pesquisados (levantamento de dados e retorno) e, então, foram informados sobre detalhes do termo de consentimento formal.

Depois desses procedimentos, os alunos foram convidados a responder ao instrumento de pesquisa com base em percepções e conhecimentos apropriados. O tempo de resposta médio de cada grupo foi de aproximadamente 10 minutos (excluído o tempo consumido com instruções detalhadas e assinatura do termo de consentimento formal).

\section{ANÁLISE DE RESULTADOS}

Ao final da coleta de dados, foram considerados apenas os questionários com consentimento formal, selecionadas as respostas válidas e transportados os dados para o sistema aplicativo SPSS ${ }^{\odot}$. Vários procedimentos foram desenvolvidos com o objetivo de encontrar as respostas para as questões de pesquisa estabelecidas.

\subsection{Testes preliminares}

Com o objetivo de conhecer os dados e selecionar os procedimentos estatísticos adequados para examinar as hipóteses apresentadas neste estudo, um conjunto de testes preliminares foi realizado. Analisando a base de dados, foi possível observar que o teste de normalidade
Kolmogorov-Smirnov (Levin e Rubin, 1997), com as variáveis dependentes deste estudo, não indicou a aproximação para uma distribuição normal. Resultados similares também foram obtidos com o teste de Shapiro-Wilk. $A$ análise de confiabilidade do instrumento de pesquisa revelou um Alfa de Cronbach de 0,929, confirmando sua consistência interna. Com base nos resultados dos testes preliminares e considerando as suposições para utilização de testes paramétricos estatísticos, as análises de dados subseqüentes foram desenvolvidas utilizando procedimentos não-paramétricos: Spearman (correlação), Mann-Whitney e Kruskal-Wallis (comparação de médias) e Análise de Conglomerados (K-means). 


\subsection{Análise estatística descritiva}

É importante lembrar que a amostra foi constituída por alunos de pós-graduação matriculados em sete cursos presenciais de MBA, bem-conceituados em suas áreas de concentração. As áreas de concentração de cada MBA eram, especificamente: Atuária $(n=13)$, Auditoria $(n=15)$, Controladoria $(n=100)$, Mercado de Capitais $(n=11)$, Controladoria na Gestão Pública $(n=22)$, Risco $(n=14)$ e Tecnologia da Informação $(n=13)$. Um dos respondentes não identificou o curso. Convém notar a preponderância de respondentes do programa de Controladoria, o que guarda relação com o tamanho do programa (maior) e idade (mais antigo).

A amostra era composta por $67 \%$ de alunos do sexo masculino e com idade média de 35, I anos (Desvio-padrão $=7,1$ ). Uma parcela expressiva (33\%) possuía, além do bacharelado, uma segunda formação (e.g., qualificação ou especialização). Um total de $56 \%$ dos sujeitos era graduado em Administração (20\%) ou em Contabilidade $(36 \%)$.

Ao se considerar a experiência em Internet desses sujeitos, os resultados da pesquisas mostraram uma utilização baixa dos recursos "Mensagens Instantâneas" e "Grupos de Discussão" (Tabela I O). Esses resultados podem ser relacionados à dificuldade de utilização desses recursos no ambiente de trabalho e estão coerentes com a média de idade dos sujeitos. O conjunto dos resultados indica que os potenciais alunos têm experiência e confiança para aprender através de um ambiente educacional on-line.

Quando considerado o aspecto de experiência em cursos on-line, foram obtidos resultados muito próximos de pessoas que possuem e não possuem qualquer experiência em e-learning. Já em relação à propensão em fazer cursos on-line, $76 \%$ da amostra respondeu positivamente à pesquisa, sendo $35 \%$ sujeitos com experiência em cursos online e 4 I \% sem experiência com cursos on-line. As porcentagens são as mesmas se observado o desejo de freqüentar, simultaneamente, cursos semi-presenciais: $76 \%$ do total da amostra. E, ao se analisarem, separadamente, os números da amostragem também são os mesmos: $35 \%$ (aqueles que já possuem experiência em e-learning) e $41 \%$ (aqueles sem experiência).

A Tabela 20 resume os atributos que influenciam a decisão de se matricular, em termos de freqüência de respostas reunidas nessa amostra. Considerando o fato de que a amostra foi constituída por alunos matriculados em programas de MBA de alto nível, as respostas revelaram uma grande preocupação em relação à instituição, conteúdo do curso e professores (incluindo interação com docentes).

Esses resultados estão consistentes com os fatores identificados em estudos anteriores que influenciariam a seleção de disciplinas em programas de nível superior por alunos que estão por concluir o segundo grau e estudantes universitários. Os estudos que analisam esses fatores (ou atributos) de cursos on-line definidos como qualidade no ensino, mensalidades, reputação, conforto, corpo docente etc., apresentaram como fatores determinantes no critério de seleção: utilidade, reputação e imagem da instituição de ensino, além dos valores do custo (Huang, 2005). É válido mencionar que não há grande divergência em relação aos fatores quando da escolha entre cursos on-line e presenciais.

Similarmente, a Tabela $3 \bullet$ apresenta as expectativas dos sujeitos quanto às soluções disponíveis nos cursos online. Tais resultados apontam a relevância dos exercícios e do suporte virtual os quais se relacionam às características da educação de adultos e à metacognição analisadas na amostra.

Ao analisar o interesse dos alunos em áreas específicas de conteúdo do curso (27 escolhas), Avaliação de Empresas (4,23, em uma escala de cinco pontos) foi a mais bem pontuada, seguida por Interpretação das Demonstrações Contábeis $(3,90)$ e Controle Gerencial $(3,89)$. As duas menos pontuadas foram Controladoria em Organizações na Área de Saúde $(2,13)$ e Contabilidade Governamental $(2,30)$.

Tabela 1 - Experiências com Internet - uso regular (freqüências)

\begin{tabular}{ll|ll} 
E-mail & $100 \%$ & Compras/vendas & $56 \%$ \\
\hline Mensag. Instantânea & $30 \%$ & Sites Governamentais & $80 \%$ \\
\hline Notícias & $88 \%$ & Downloads & $61 \%$ \\
\hline Serviços Bancários & $82 \%$ & Grupos de Discussão & $23 \%$ \\
\hline Mecanismo pesquisa & $96 \%$ & \multicolumn{2}{l}{}
\end{tabular}

Tabela 2 | Atributos que influenciam a decisão de matrícula (freqüências)

\begin{tabular}{ll|ll} 
Instituição & $91 \%$ & Tecnologia & $51 \%$ \\
\hline Conteúdo do Curso & $82 \%$ & Duração do curso & $49 \%$ \\
\hline Interação com docentes & $72 \%$ & Tempo Extra-Classe & $39 \%$ \\
\hline Corpo docente & $64 \%$ & Interação com colegas & $39 \%$ \\
\hline Material de instrução & $68 \%$ & Opções de pagamento & $27 \%$ \\
\hline Custo & $58 \%$ & &
\end{tabular}


Tabela 3 Expectativa a respeito de soluções on-line (freqüências)

\begin{tabular}{ll|ll} 
Exercícios On-line & $82 \%$ & Suporte Telefônico & $47 \%$ \\
\hline Suporte & $78 \%$ & Chat de Texto & $43 \%$ \\
\hline Fórum de discussão & $62 \%$ & Apresentação de Slides & $35 \%$ \\
\hline Aula-Chat de Voz & $55 \%$ & Animações & $15 \%$ \\
\hline Aula-Chat de Texto & $49 \%$ & PDA \& Telefone celular & $19 \%$ \\
\hline Apresentação com Voz & $48 \%$ & \multicolumn{2}{l}{}
\end{tabular}

\subsection{Correlações}

Para analisar as correlações entre os interesses dos sujeitos nos diversos conteúdos, todos os 27 cursos foram agrupados em sete áreas. O agrupamento foi feito considerando-se a estrutura do exame CPA nos Estados Unidos (AICPA, 2002), o currículo básico da National Business Education Association (NBEA, 200I), os objetivos educacionais da contabilidade (Accounting Education Change Commission - AECC, 1990), bem como elementos da certificação de profissionais no Brasil. A Tabela $4 \mathbf{O}$ apresenta a estrutura envolvendo áreas e conteúdos dos cursos.
Após a condução da análise de correlações, baseada nessas áreas (Tabela 5 O) foi possível observar que a área Contabilidade Financeira $\left(A_{1}\right)$, apresenta correlações significativas altas com quase todas as outras áreas. A correlação significativa mais alta é entre Contabilidade Financeira e $\left(A_{1}\right)$ e Contabilidade Gerencial $\left(A_{4}\right)$.

\subsection{Comparação de Médias}

Para se testar a existência de diferenças significativas entre as preferências das 27 áreas de conteúdo e as propensões dos alunos em freqüentar ou não cursos on-line,

Tabela 4 Grupos de conteúdo do curso (Áreas)

\begin{tabular}{|c|c|}
\hline Áreas & Cursos \\
\hline $\mathrm{A}_{1}-$ Contabilidade Financeira & $\begin{array}{l}\text { Contabilidade Básica, Demonstração do Fluxo de Caixa e } \\
\text { Demonstração das Origens e Aplicações de Recursos, } \\
\text { Demonstração do Valor Adicionado, Interpretação das Demonstrações Contábeis, Avaliação de Em- } \\
\text { presas, Contabilidade Internacional, } \\
\text { Conversão das Demonstrações Contábeis para Moeda Estrangeira, Contabilidade de Custo, } \\
\text { ABC,Contabilidade Bancária,Contabilidade Ambiental }\end{array}$ \\
\hline $\mathrm{A}_{2}-$ Tributação & Contabilidade Tributária, Planejamento Tributário \\
\hline $\mathrm{A}_{3}-$ Cont. Governamental & Contabilidade Governamental, Contabilidade do Terceiro Setor \\
\hline $\mathrm{A}_{4}-$ Contabilidade Gerencial & Orçamento, Controle Gerencial, Controladoria em organizações da Área de Saúde \\
\hline $\begin{array}{l}A_{5}-\text { Finanças, Mercado } \\
\text { Financeiro e Governança }\end{array}$ & $\begin{array}{l}\text { Finanças, Mercado Financeiro, } \\
\text { Análise Financeira, Derivativos } \\
\text { Governança Corporativa }\end{array}$ \\
\hline$A_{6}-$ Habilidades Correlatas & Matemática Financeira, Estatística Aplicada \\
\hline$A_{7}-$ Jogos de empresas & Jogos de Bancos, Jogos de Risco \\
\hline
\end{tabular}

Tabela 5 || Correlações (Spearman)

\begin{tabular}{|c|c|c|c|c|c|c|c|}
\hline & $A_{1}$ & $A_{2}$ & $A_{3}$ & $A_{4}$ & $A_{5}$ & $A_{6}$ & $A_{7}$ \\
\hline$A_{1}$ & 1,000 & & & & & & \\
\hline$A_{2}$ & $0,557 * *$ & 1,000 & & & & & \\
\hline $\mathrm{A}_{3}$ & $0,601 * *$ & $0,477 * *$ & 1,000 & & & & \\
\hline $\mathrm{A}_{4}$ & $0,703 * *$ & $0,426 * *$ & $0,591 * *$ & 1,000 & & & \\
\hline $\mathrm{A}_{5}$ & $0,601 * *$ & $0,454^{* *}$ & $0,346 * *$ & $0,426 * *$ & 1,000 & & \\
\hline$A_{6}$ & $0,450 * *$ & $0,229 * *$ & $0,346 * *$ & $0,419 * *$ & $0,429 * *$ & 1,000 & \\
\hline$A_{7}$ & $0,292 *$ & $0,393 * *$ & $0,185^{*}$ & $0,186 * *$ & $0,519 * *$ & $0,321 * *$ & 1,000 \\
\hline
\end{tabular}


foi realizado o teste não-paramétrico de Mann-Whitney. Os resultados desse teste revelaram apenas duas diferenças: Contabilidade Básica $(U=2489,5, Z=-2,562$, sig. $=$ $0,010)$, e Contabilidade Internacional $(U=2608,0, Z=$ $-2,189$, sig. $=0,029$ ). Realizando o mesmo teste novamente, mas considerando os conteúdos agrupados em sete áreas, não foram encontradas diferenças significativas em termos da propensão aos cursos on-line.

$A$ base de dados também foi testada para verificar se existiam diferenças significativas entre as preferências dos cursos quando considerado o gênero de cada aluno. O teste de Mann-Whitney revelou diferenças significativas na escolha de homens e mulheres nas seguintes áreas de conteúdo: Avaliação de Empresas $(U=33 \mid 4,5, Z=-2,101$, sig. $=0,036)$, Contabilidade Governamental $(U=3358,0$, $Z=-1,805$, sig. $=0,071)$, Interpretação das Demonstrações Financeiras $(U=3285,0, Z=-1,999$, sig. $=0,046)$, Derivativos $(U=3333,5, Z=-1,839$, sig. $=0,066)$, Contabilidade Ambiental $(U=3342,0, Z=-1,815$, sig. $=$ $0,070)$, Estatística Aplicada $(U=3288,0, Z=-1,971$, sig. $=0,049)$ e Jogos de Risco $(U=3294,0, Z=-1,961$, sig. $=0,050)$. Ao se realizar o mesmo teste novamente, mas agora, considerando o conteúdo agrupado em sete áreas, apenas Jogos de Empresas apresentou diferença significativa quanto à disposição em freqüentar cursos on-line $(U=3365,5, Z=-1,731$, sig. $=0,083)$.

Ao se compararem preferências e experiência acadêmica, utilizando o teste de Kruskal-Wallis, sete áreas de conteúdo apresentaram diferenças significativas: Demonstração do Fluxo de Caixa $\left(\div^{2}=8,688\right.$, sig. $\left.=0,034\right)$, Contabilidade Internacional $\left(\div^{2}=12,792\right.$, sig. $\left.=0,005\right)$, Conversão das Demonstrações Contábeis para Moeda Estrangeira $\left(\div^{2}=15,531\right.$, sig. $\left.=0,001\right)$, Orçamento $\left(\div^{2}=10,426\right.$, sig. $=0,015)$, Contabilidade de Custos $\left(\div^{2}=8,699\right.$, sig. $=$ $0,034)$, Activity Based Costing $\left(\div^{2}=8,499\right.$, sig. $\left.=0,037\right) \mathrm{e}$ Jogos de Risco $\left(\div^{2}=6,389\right.$, sig. $\left.=0,094\right)$.

A comparação entre preferências por cursos e a experiência dos sujeitos em educação on-line, (teste de MannWhitney), mostrou que apenas uma área de conteúdo apresentava diferença significativa: Contabilidade Básica $(\mathrm{U}=3742,5, \mathrm{Z}=-1,755$, sig. $=0,079)$.

O teste de Kruskall-Wallis, considerando as áreas de concentração dos cursos (e.g., MBA em Controladoria, Risco, TI, Mercado de Capitais etc.) e o interesse por conteúdos de cursos específicos indicou que apenas o tema de Governança Corporativa não apresentou diferença significativa $\left(\div^{2}=5,555\right.$, sig. $\left.=0,475\right)$. Para todos os demais, foi observada diferença significativa. Na utilização do teste de Kruskall-Wallis, com os conteúdos agregados por áreas, considerando os grupos de alunos e a propensão à participação em cursos on-line, não foram encontradas diferenças significativas.

Quando comparado os alunos do MBA em Controladoria $(n=100)$ com os alunos dos demais cursos $(n=88)$ e efetuado o teste de Mann-Whitney, os seguintes temas não apresentaram diferenças significativas: Demonstração do Fluxo de Caixa e Demonstração das Origens e Aplica- ções de Recursos $(U=4368,5, Z=-0,086$, sig. $=0,93 \mathrm{I})$, Orçamento ( $U=3885,5, Z=-1,442$, sig. $=0,149)$, Controladoria em Organizações da Área de Saúde $(U=4 \mid$ I 9,5, $Z=-0,802$, sig. $=0,422$ ), Contabilidade Governamental $(\mathrm{U}=4040,0, Z=-1,014$, sig. $=0,311)$, Contabilidade do Terceiro Setor $(U=4295,0, Z=-0,290$, sig. $=0,772)$, Contabilidade Bancária $(U=3905,0, Z=-1,358$, sig. $=$ $0,174)$, Mercado Financeiro $(U=4105,5, Z=-0,827$, sig. $=0,408)$, Interpretação das Demonstrações Contábeis $(U=4376,0, Z=-0,067$, sig. $=0,947)$, Derivativos $(U=$ 4278,0, $Z=-0,336$, sig. $=0,737$ ), Governança Corporativa $(U=3997,0, Z=-1,142$, sig. $=0,254)$, Matemática Aplicada ( $U=4160,5, Z=-0,663$, sig. $=0,508)$, Jogos de Bancos $(U=4 \mid 44,0, Z=-0,704$, sig. $=0,482)$ e Jogo de Risco $(U=3871,0, Z=-1,463$, sig. $=0,143)$.

Quando considerados os cursos agrupados em áreas, os resultados revelaram diferenças significativas em Contabilidade Governamental $(U=4105,5, Z=-0,804$, sig. $=$ $0,42 \mathrm{I})$, Contabilidade Gerencial $(U=4140,5, Z=-0,701$, sig. $=0,483)$ e Finanças, Mercado Financeiro e Governança Corporativa $(U=4275,0, Z=-0,337$, sig. $=0,736)$.

\subsection{Análise de conglomerados}

Foi conduzida uma análise de conglomerados (K-means, método interação e classificação) para verificar as características dos estudantes e suas preferências por áreas de conteúdo específicas. Os resultados obtidos no estudo indicaram que, analisando os estudantes em termos de seu interesse em freqüentar cursos específicos (considerando toda a amostra), foi possível identificar dois grupos (estatisticamente distintos). No grupo $C_{\text {, }}$ (baixos níveis de preferência), com 6 I sujeitos, as maiores preferências 3,43 (Avaliação de Empresas) e as menores preferências 1,20 (Controladoria em Organizações na Área de Saúde). E, no grupo $\mathrm{C}_{2}$ (maiores níveis de preferência, ou "entusiastas"), com 128 sujeitos, no qual os maiores e os menores índices eram respectivamente, 4,61 (Avaliação de Empresas) e 2,57 (Controladoria em Organizações na Área de Saúde).

Se analisadas as características específicas desses grupos, o grupo C, possui $60 \%$ das pessoas com propensão para freqüentar cursos on-line, $67 \%$ dos estudantes do sexo masculino, $77 \%$ com graduação completa, $22 \%$ com especialização (certificado) e $36 \%$ que afirmavam possuir experiência com cursos on-line. O grupo $\mathrm{C}_{2}$ ("entusiastas"), por outro lado, apresentava $77 \%$ de pessoas com propensão para freqüentar cursos on-line, $66 \%$ de alunos do sexo masculino, $61 \%$ com bacharelado, $37 \%$ com especialização (certificado), e 46\% com experiência prévia em cursos on-line.

As correlações baseadas no grupo $C_{2}(n=128)$ são muito consistentes em termos de resultados da Tabela 5 (ver Tabela 7 ), com exceção das correlações negativas encontradas entre Contabilidade Governamental $\left(A_{3}\right)$ e Jogos de Empresas $\left(A_{7}\right)$ e entre Contabilidade Gerencial $\left(A_{4}\right)$ e Jogos de Empresas $\left(A_{7}\right)$. O grupo $C_{1}(n=61)$ apresenta correlações significativas interessantes: existe uma forte correlação positiva entre Contabilidade Financeira $\left(A_{1}\right)$, 
Contabilidade Governamental $\left(A_{3}\right)$ e Contabilidade Gerencial $\left(A_{4}\right)$; e existem correlações baixas ou negativas entre Jogos de Empresas $\left(A_{7}\right)$ e Contabilidade Financeira $\left(A_{1}\right)$, Contabilidade Governamental $\left(A_{3}\right)$, Contabilidade Gerencial $\left(A_{4}\right)$ e Habilidades Correlatas $\left(A_{6}\right)$, como mostrado na Tabela 60.

No entanto, apesar de não conclusivos, pode-se colocar que os resultados reforçam a idéia de que é relevante a divisão encontrada em termos das preferências de conteúdo, porém ambos os grupos possuem alta porcentagem de estudantes com propensão a freqüentar cursos on-line. Além disso, os resultados indicam que, entre os "entusiastas”, maior proporção de estudantes com formação acadêmica (especialização) e experiência em cursos on-line que se interessa por educação a distância. No entanto, com relação a gênero, os dois grupos apresentam proporções semelhantes de representantes do sexo masculino, inclusive próximas à proporção de homens da amostra total, o que contraria resultados de pesquisas anteriores. Porém, o contexto sócio-econômico-cultural e da área profissional dos cursos considerados podem ter influenciado esses resultados. Cabe ressaltar que a amostra representa observações empíricas de proporção de estudantes participantes de programas e cursos dessa natureza principalmente no que diz respeito à distribuição entre homens e mulheres. Esse fator deve ser levado em consideração ao serem analisados os resultados aqui apresentados. Uma experiência prévia em e-learning não parece tão relevante nessa análise. De forma isolada, nenhuma das sete áreas curriculares mostrou influências expressivas como determinantes para grupos.

Tabela 6 - Correlações (Spearman) - Grupo $C_{1}$

\begin{tabular}{c|c|c|c|c|c|c|c} 
& $\mathbf{A}_{\mathbf{1}}$ & $\mathbf{A}_{\mathbf{2}}$ & $\mathbf{A}_{\mathbf{3}}$ & $\mathbf{A}_{\mathbf{4}}$ & $\mathbf{A}_{\mathbf{5}}$ & $\mathbf{A}_{\mathbf{6}}$ & $\mathbf{A}_{\mathbf{7}}$ \\
$\mathrm{A}_{1}$ & 1,000 & & & & & & \\
\hline $\mathrm{A}_{2}$ & $0,327^{*}$ & 1,000 & & & & & \\
\hline $\mathrm{A}_{3}$ & $0,360^{* *}$ & 0,042 & 1,000 & & & & \\
\hline $\mathrm{A}_{4}$ & $0,477^{* *}$ & $-0,009$ & 0,165 & 1,000 & & & \\
\hline $\mathrm{A}_{5}$ & $0,146^{* *}$ & 0,022 & 0,154 & 0,082 & 1,000 & & \\
\hline $\mathrm{A}_{6}$ & $-0,023^{* *}$ & $-0,119$ & 0,050 & 0,029 & $0,372^{* *}$ & 1,000 & \\
\hline $\mathrm{A}_{7}$ & $0,051^{*}$ & $0,323^{*}$ & 0,141 & $-0,095$ & $0,364^{* *}$ & $0,313^{*}$ & 1,000 \\
& *-correlação significativa considerando o nível de 0,05 \\
**- correlação significativa considerando o nível de 0,01
\end{tabular}

Tabela 7 Correlações (Spearman) - Grupo $C_{2}$

\begin{tabular}{c|c|c|c|c|c|c|c} 
& $\mathbf{A}_{\mathbf{1}}$ & $\mathbf{A}_{\mathbf{2}}$ & $\mathbf{A}_{\mathbf{3}}$ & $\mathbf{A}_{\mathbf{4}}$ & $\mathbf{A}_{\mathbf{5}}$ & $\mathbf{A}_{\mathbf{6}}$ & $\mathbf{A}_{\mathbf{7}}$ \\
$\mathrm{A}_{1}$ & 1,000 & & & & & & \\
\hline $\mathrm{A}_{2}$ & $0,278^{* *}$ & 1,000 & & & & & \\
\hline $\mathrm{A}_{3}$ & $0,447^{* *}$ & $0,381^{* *}$ & 1,000 & & & & \\
\hline $\mathrm{A}_{4}$ & $0,438^{* *}$ & 0,159 & $0,542^{* *}$ & 1,000 & & & \\
\hline $\mathrm{A}_{5}$ & $0,320^{* *}$ & $0,262^{* *}$ & 0,071 & 0,048 & 1,000 & & \\
\hline $\mathrm{A}_{6}$ & $0,379^{* *}$ & 0,133 & $0,319^{* *}$ & $0,329^{* *}$ & $0,274^{* *}$ & 1,000 & \\
\hline $\mathrm{A}_{7}$ & 0,074 & $0,216^{*}$ & $-0,28$ & $-0,035$ & $0,432^{* *}$ & 0,058 & 1,000 \\
\multicolumn{8}{c}{ *- correlação significativa considerando o nível de 0,05} \\
**-correlação significativa considerando o nível de 0,01
\end{tabular}

\section{CONCLUSÕES E RECOMENDAC̣ÕES}

A educação on-line pode oferecer apoio à reflexão sobre o atual estágio da educação em negócios em termos de alternativas instrucionais e oportunidades de soluções confiáveis e apropriadas, visando a um público específico de alunos adultos trabalhadores envolvidos com as organizações, que trazem experiência profissional e estão interessados em perseguir objetivos educacionais mais avançados (e.g., pro- gramas de pós-graduação). Nesse sentido, o estudo pôde coletar evidências para sustentar essa discussão envolvendo as duas dimensões da educação superior (desenvolvimento de recursos humanos e educação em contabilidade) ao responder às perguntas acima mencionadas, baseadas em uma amostra de 189 alunos de programas presenciais de MBA em Contabilidade e áreas afins no Brasil. 
Considerando a questão $\mathrm{Q}$, da pesquisa, os cursos mais bem pontuados, portanto com maior propensão, foram Avaliação de Empresas (4,23, em uma escala de cinco pontos) seguido por Interpretação das Demonstrações Contábeis $(3,90)$ e Controle Gerencial $(3,89)$. Os cursos menos pontuados foram Controladoria em Organizações na Área de Saúde $(2,13)$ e Contabilidade Governamental $(2,30)$. Os resultados da análise mostram a preferência por cursos fortemente relacionados à tomada de decisão em temas relevantes da contabilidade. Os cursos que despertam menos interesse estão diretamente ligados a temas bastante específicos da área. Esses resultados poderiam ser explicados pela proporção de participantes da pesquisa que tenham relação com essas áreas específicas (e.g. Atuária e Controladoria na Gestão Pública). Outras pesquisas com proporções diferentes de sujeitos podem confirmar ou não essa conclusão.

A questão $Q_{2}$ analisa se existem correlações significativas em termos de preferências por determinadas áreas de concentração. As correlações são todas significativas. Porém as correlações mais altas são encontradas com a área de Contabilidade Financeira. Por outro lado, as menores correlações foram encontradas com as áreas $A 6$ e $A 7$ (Habilidades Correlatas e Jogos). A correlação alta percebida entre as principais áreas de concentração do estudo da Contabilidade pode talvez indicar que os cursos são percebidos como possibilidades de especialização profissional e aprofundamento, mais do que como oportunidade de extensão ou formação geral.

Se considerada a questão $Q_{3}$, pode-se constatar pontos a serem destacados em termos de: (a) gênero, (b) formação acadêmica; (c) propensão para freqüentar cursos on-line e (d) experiência em e-learning. No caso de gênero foram encontradas diferenças significativas em sete cursos (Avaliação de Empresas, Contabilidade Governamental, Interpretação das Demonstrações Financeiras, Derivativos, Contabilidade Ambiental, Estatística Aplicada e Jogos de Risco) e, quando agrupados em áreas, em apenas um caso (Jogos de Empresas). Em termos de experiência acadêmica foram encontradas diferenças significativas em sete cursos (Demonstração do Fluxo de Caixa e Demonstração das Origens e Aplicações de Recursos, Contabilidade Internacional, Orçamento, Conversão das Demonstrações Contábeis para Moeda Estrangeira, Contabilidade de Custos, Activity Based Costing e Jogos de Risco). Em relação à experiência em educação on-line apenas um curso mostrou diferença significativa (Contabilidade Básica). Embora não haja meios de se encontrar um padrão para esses resultados, evidencia-se que as preferências dos estudantes para cursos específicos são diferentes em alguns casos, revelando que o conteúdo possui grande influência em algumas decisões (e. g., matrícula em cursos on-line) que devem ser verificadas mais detalhadamente.

$\mathrm{Na}$ análise da questão $\mathrm{Q}_{4}$, se segregados os alunos entre as concentrações (MBA em Controladoria vs MBAs em áreas afins), os resultados não mostraram diferenças significativas em 13 dos 27 cursos. Ao serem analisados os conteúdos dos cursos, conclui-se que se tratam de cur- sos básicos que interessam a todos, ou de cursos bastante específicos. Quando analisados os cursos agrupados por áreas foram encontradas diferenças significativas nas áreas de Contabilidade Governamental, Contabilidade Gerencial, Finanças, Mercado Financeiro e Governança Corporativa.

Quanto à questão $\mathrm{Q}_{5}$, pôde ser observada, por meio da análise de conglomerados, a existência de dois agrupamentos de alunos definidos $\left(\mathrm{C}_{1}\right.$ - de menor preferência e $\mathrm{C}_{2}$ - de maior preferência, ou "entusiastas"), ambos com altas porcentagens de estudantes que pretendem freqüentar cursos on-line (respectivamente $60 \%$ e $77 \%$ ). As características dos grupos permitiram perceber entre os "entusiastas" um percentual maior de pessoas com experiência em cursos on-line e com formação acadêmica em nível de especialização. Não há diferença em termos de gênero, porém cabe ressaltar que observações empíricas de proporção de estudantes participantes de programas e cursos dessa natureza, principalmente, no que diz respeito à distribuição entre homens e muIheres. Quanto à análise de correlação cabe ressaltar que o grupo C, apresenta correlações significativas interessantes: uma forte correlação positiva entre Contabilidade Financeira $\left(A_{1}\right)$, Contabilidade Governamental $\left(A_{3}\right)$ e Contabilidade Gerencial $\left(A_{4}\right)$; e correlações baixas ou negativas entre Jogos de Empresas $\left(A_{7}\right)$ e Contabilidade Financeira $\left(A_{1}\right)$, Contabilidade Governamental $\left(A_{3}\right)$, Contabilidade Gerencial $\left(A_{4}\right)$ e Habilidades Correlatas $\left(A_{6}\right)$. Em relação ao grupo $C_{2}$, as correlações são bastante consistentes com as encontradas quando considerada a amostra global.

Ademais, essas evidências e conclusões embasam a idéia de que o conteúdo do curso possui papel relevante na tomada de decisão dos estudantes de freqüentar ou não cursos on-line, especialmente no que se refere à contabilidade. Essa particularidade requer uma atenção maior de acadêmicos para que se ofereça aos tomadores de ações detalhes específicos (e.g., instituições, faculdades, estudantes e outros provedores de tecnologia da educação). De acordo com a experiência adquirida nesse trabalho, uma futura pesquisa na área considerará aspectos como (a) natureza dos cursos (cursos com enfoque mais prático ou mais teórico); (b) relevância de conteúdo; (c) dimensionamento da carga de trabalho; (d) tendência ao desenvolvimento de docentes ou tutores para o desenvolvimento dos cursos e (e) estratégias de educação.

Duas recomendações baseadas nessas reflexões ficam registradas. Primeiro, é importante que os fornecedores de soluções educacionais considerem as principais áreas de conteúdo como prioridade (educação on-line não pode ser considerada como meio de lidar as áreas de importância secundária). Segundo, visando ao desenvolvimento profissional dos recursos humanos, os resultados revelaram que deve ser dada grande importância ao estudante. Portanto, ajudá-los em aspectos como metacogniç̧ão e considerar suas preferências em termos de conteúdo é o principal componente de decisão em termos de alternativas de educação on-line (incluindo soluções semipresenciais, ao se considerar que $76 \%$ se matriculariam simultaneamente em cursos presenciais e on-line). 


\section{CONTRIBUIC̣ÕES}

Considerando a discussão precedente, os resultados dessa pesquisa podem oferecer contribuições iniciais para diminuir a distância entre os campos de desenvolvimento de recursos humanos e educação contábil, ambos buscando soluções educacionais para uma categoria profissional específica, baseada em relevantes dimensões: (a) planejar o ambiente da educação on-line; (b) desenvolver e ajustar o currículo às estratégias instrucionais, (c) apoiar o desenvolvimento da metacognição alunos adultos trabalhadores e (d) combinar o ambiente de trabalho com as necessida- des individuais na formatação de um programa instrucional. Além disso, de acordo com a idéia de Gall, Gall e Borg (2003, p .9), que "uma pesquisa não poderá provar uma teoria, mas embasá-la", ao longo deste estudo foram considerados elementos e argumentos para analisar o relacionamento entre conteúdo de curso e propensão de alunos de pós-graduação em contabilidade para cursos on-line. Nesse sentido, este estudo contribui com a área trazendo algumas evidências nesses aspectos particulares.

\section{Referências}

ACCOUNTING EDUCATION CHANGE COMMISSION [AECC]. Objectives of education for accountants: Position statement number one. Issues in Accounting Education, v. 5, n. 2, p. 307-312, 1990

ALESSI; S. M.; TROLLIP, S. R. Multimedia for learning: methods and development. 3 ed. Needham Heights, MA: Allyn and Bacon, 2001.

AMERICAN INSTITUTE OF CERTIFIED PUBLIC ACCOUNTANTS [AICPA]. Information for uniform CPA examination candidates. New York: AICPA, 2002.

ARBAUGH, J. B. An exploratory study of the effects of gender on student learning and class participation in an Internet-based MBA course. Management Learning, v. 31, n. 4, p. 503-519, 2000a.

; HWANG, A. Does 'teaching presence' exist in on-line MBA courses? The Internet and Higher Education, n. 9, p. 9-21, 2006.

. How much does "subject matter" matter? A study of disciplinary effects in on-line MBA courses. Academy of Management Learning \& Education, v. 4; n. 1, p. 57-73, 2005

;DURAY R. Technological and structural characteristics, student learning and satisfaction with web-based courses: an exploratory study of two on-line MBA programs. Management Learning, v. 33, n. 3, p. 331-347, 2002.

. Virtual classroom characteristics and student satisfaction with Internet-based MBA courses. Journal of Management Education v. 24, n. 1, p. $32-35,2000$ b.

BALL, S. R. The role of organizational culture in innovation adoption: teaching through the internet in specialized schools of business, $\mathrm{PhD}$ Dissertation, University of Michigan, 2005.

BROWER, H. H. On emulating classroom discussion in a distance-delivered OBHR course: creating an on-line learning community. Academy of Management Learning and Education, v. 2, n. 1, p. 22-36, 2003.

BRYANT, S. M.; KAHLE, J. B.; SCHAFER, B. A. Distance education: a review of the contemporary literature. Issues in Accounting Education, v. 20, n. 3, p. 255-272, 2005.

CRESWELL, J. W. Research design: Qualitative, quantitative, and mixed methods approaches. 2 ed. Thousand Oaks: Sage Publications, 2003

CUMMINGS, T. G.; WORLEY, C. G. Organization development and change. 8 ed. Mason: South-Western/Thomson, p. 396-433, 2005.

DABBAGH, N.; BANNAN-RITLAND, B. On-line learning: concepts, strategies, and application. Upper Saddle River: Pearson Education, 2005.

DUNBAR, A. E. Genesis of an on-line course, Issues in Accounting Education, v. 19, n. 3, p. 321-343, 2004.

FODOR, J. T. On-line college courses: great for some people - not so great for others. Promotion \& Education, v. 10, n. 2, p. $72,2003$.

GAGNE, M.; SHEPERD, M. A comparison between a distance and a traditional graduate accounting class. THE Journal, v. 28, n. 9, p. 6, 2001. GALL, M. D.; GALL, J. P.; BORG, W. R. Educational research: an introduction. 7 ed. Boston: Allyn \& Bacon, 2003.

GALLAHER, Jr., J. W. The adoption of e-learning across professional groups in a fortune 500 company, PhD Dissertation, University of Illinois at Urbana-Champaign, 2002

HOCHBERG, J. On-line MBA programs: emulating global búsiness, Distance Education Report. Magna, 2006.

HUANG, C. H. Exploring employed-learner's choice profiles for VMBA programs. Journal of American Academy of Business, v. 7, n. 2, p. 203$211,2005$.

KIM, K-J, LIU, S. E BONK, C. J. On-line MBA students' perceptions of on-line learning: benefits, challendes; and suggestions, The Internet and Higher Education, n. 8, p. 335-344, 2005.

LAVOIE, D.; ROSMAN, A. J. Using active student-centered learning-based instructional design to develop faculty and improve course design, delivery, and evaluation, Issues in Accounting Education, v. 22, n. 1, p. 105-118, 2007. 
LEVIN, R. I.; RUBIN, D. S. Statistics for management. 7 ed. Upper Saddle River, NJ: Prentice Hall, 1997.

McKEACHIE; W. J. McKeachie's teaching tips: strategies, research, and theory for college and university teachers. 11 ed. New York, NY: Houghton Mifflin, 2002.

MERRIAM, S. B.; CAFFARELLA, R. S. Learning in adulthood: a comprehensive guide. 2 ed. San Francisco, CA: Jossey-Bass, 1999.

NATIONAL BUSSINESS EDUCATION ASSOCIATION National Standards for Business Education. Reston: NBEA, 2001.

NOBLE, D. F. Digital diploma mills: the automation of higher education. New York: Monthly Review Press, 2002.

PAISEY, C.; PAISEY, N. An analysis of accounting education research in accounting education: An international journal: 1992-2001. Accounting Education, v. 13, n. 1, p. 69-99, 2004.

REA, L. M.; PARKER, R. A. Designing and conducting survey research. 2 ed. San Francisco: Jossey-Bass, 1997.

ROMISZOWSKI, A. J. How's the e-learning baby? Factors leading to success or failure of an educational technology innovation, Educational Technology, v. 44, n. 1, p. 4-27, 2004.

SCHRUM, L.; BENSON, A. On-line professional education: a case study of an MBA program through its transition to an on-line model. Journal of Asynchronous Learning Networks, v. 4, n. 1, p. 52-61,2000.

SMITH, P. A. Understanding self-regulated learning and its implications for accounting educators and researchers, Issues in Accounting Education, v. 16, n. 4, p. 663-700, 2001.

SWANSON, R. A.; HOLTON, III, E. F. Foundations of human resource development. San Francisco: Berrett-Koehler, 2001.

TALLENT-RUNNELS et al. Teaching courses on-line: a review of the research, Review of Educational Research, v. 76, n. 1, p. 93-135, 2006.

TOOLEY, J. The Global Education Industry: lessons from private education in developing countries. IEA Studies in Education, n. 7, Washington: IFC, 1999.

WALSH, D. On-line MBA, Training and Development, p. 108-109, August 2006.

WILSON, R. Accounting education research: A retrospective over ten years with some pointers to the future. Accounting Education, v. 11, n. 4 , p. 295-310, 2002.

YANG, B.; LU, D. R. Predicting academic performance in management education: an empirical investigation of MBA success, Journal of Education for Business, v. 77, n. 1, p. 15-20,2001.

\section{NOTA - Endereço dos autores}

Universidade de São Paulo

Faculdade de Economia, Administração e Contabilidade

Departamento de Contabilidade e Atuária

Av. Prof. Luciano Gualberto, 908 - prédio 3 - Cidade Universitária

São Paulo - SP

05508-900 